\title{
Veterinary Immunology and Immunopathology
}

\author{
journal homepage: www.elsevier.com/locate/vetimm
}

\section{Camelid immunoglobulins and nanobody technology}

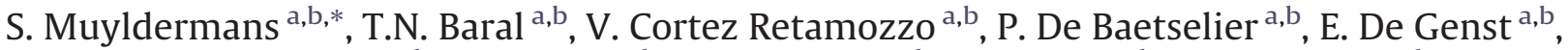 \\ J. Kinne ${ }^{c}$, H. Leonhardt ${ }^{\mathrm{d}, \mathrm{e}}$, S. Magez ${ }^{\mathrm{a}, \mathrm{b}}$, V.K. Nguyen ${ }^{\mathrm{a}, \mathrm{b}}$, H. Revets ${ }^{\mathrm{a}, \mathrm{b}}$, U. Rothbauer ${ }^{\mathrm{d}, \mathrm{e}}$, \\ B. Stijlemans ${ }^{\mathrm{a}, \mathrm{b}}$, S. Tillib ${ }^{\mathrm{f}}$, U. Wernery ${ }^{\mathrm{c}}$, L. Wyns ${ }^{\mathrm{a}, \mathrm{b}}$, Gh. Hassanzadeh-Ghassabeh ${ }^{\mathrm{a}, \mathrm{b}}$, \\ D. Saerens ${ }^{a, b}$
}

${ }^{a}$ Laboratory of Cellular and Molecular Immunology, Vrije Universiteit Brussel, Pleinlaan 2, 1050 Brussels, Belgium

${ }^{\mathrm{b}}$ Department of Molecular and Cellular Interactions, VIB, Vrije Universiteit Brussel, Brussels, Belgium

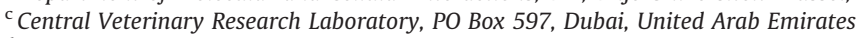

${ }^{\mathrm{d}}$ Ludwig Maximilians University Munich, Department of Biology, 82152 Planegg-Martinsried, Germany

${ }^{\mathrm{e}}$ Munich Center for Integrated Protein Science, CiPS ${ }^{M}$, Germany

${ }^{\mathrm{f}}$ Institute of Gene Biology of the Russian Academy of Sciences, Vavilov Str 34/5, 119334 Moscow, Russia

\section{A R T I C L E I N F O}

Keywords:

Dromedary

Llama

Heavy-chain antibody

Single-domain antibody

\begin{abstract}
A B S T R A C T
It is well established that all camelids have unique antibodies circulating in their blood. Unlike antibodies from other species, these special antibodies are devoid of light chains and are composed of a heavy-chain homodimer. These so-called heavy-chain antibodies (HCAbs) are expressed after a V-D-J rearrangement and require dedicated constant $\gamma$-genes. An immune response is raised in these so-called heavy-chain antibodies following classical immunization protocols. These HCAbs are easily purified from serum, and the antigenbinding fragment interacts with parts of the target that are less antigenic to conventional antibodies. Since the antigen-binding site of the dromedary HCAb is comprised in one single domain, referred to as variable domain of heavy chain of $\mathrm{HCAb}(\mathrm{VHH})$ or nanobody $(\mathrm{Nb})$, we designed a strategy to clone the $\mathrm{Nb}$ repertoire of an immunized dromedary and to select the Nbs with specificity for our target antigens. The monoclonal Nbs are well produced in bacteria, are very stable and highly soluble, and bind their cognate antigen with high affinity and specificity. We have successfully developed recombinant Nbs for research purposes, as probe in biosensors, to diagnose infections, and to treat diseases like cancer or trypanosomosis.
\end{abstract}

(c) 2008 Elsevier B.V. All rights reserved.

\section{Occurrence of heavy-chain antibodies in camelids}

Antibodies throughout mammalian species are composed of two identical $\mathrm{H}$-chains and two identical L-chains (Fig. 1A). The IgG antibodies from species of camelidae (i.e. Camelus dromedarius, Camelus bactrianus, Lama glama, Lama guanoco, Lama alpaca and Lama vicugna) form a surprising

\footnotetext{
* Corresponding author at: Laboratory of Cellular and Molecular Immunology, Vrije Universiteit Brussel, Pleinlaan 2, 1050 Brussels, Belgium. Tel.: +32 262919 69; fax: +32 26291981 .

E-mail address: svmuylde@vub.ac.be (S. Muyldermans).
}

exception to this paradigm as their serum contains also a considerable fraction of heavy-chain antibodies (HCAbs), that lack the L-chain (Hamers-Casterman et al., 1993). The $\mathrm{H}$-chain within the HCAbs is composed of three instead of four globular domains (Fig. 1A). The two constant domains are highly homologous to the Fc domains $(\mathrm{CH} 2-\mathrm{CH} 3)$ of classical antibodies (Nguyen et al., 1999; Woolven et al., 1999). The domain corresponding to the $\mathrm{CH} 1$ domain of classical antibodies is missing in HCAbs. Hence, the antigenbinding fragment of a classical antibody, the Fab, is reduced to a single variable domain in the HCAb (Fig. 1A). This variable domain referred to as $\mathrm{VHH}$ is adapted to become functional in antigen binding in absence of a variable light 
(VL) chain domain (Muyldermans et al., 1994; Vu et al., 1997; Harmsen et al., 2001; Maass et al., 2007). It has been repeatedly demonstrated that the $\mathrm{VHH}$, cloned and expressed in bacteria, is a strictly monomeric, singledomain antigen-binding entity (Muyldermans and Lauwereys, 1999). The publicity around nanotechnology and the prolate shape (i.e. shaped like a rugby ball) in the nm range (diameter of $\sim 2.5 \mathrm{~nm}$ and height of $\sim 4 \mathrm{~nm}$ ) of the $\mathrm{VHH}$ stimulated Ablynx - the company focusing on therapeutic applications of camelid antibodies - to rename VHH's as nanobodies (Nbs).

The organization is remarkably similar between the variable domain of the $\mathrm{H}$-chain of a classical antibody and that of an HCAb, the $\mathrm{VH}$ and $\mathrm{VHH}$, respectively. Nevertheless the $\mathrm{VH}$ and $\mathrm{VHH}$ contain minor, but important differences that explain the antigen-binding capacity of the VHH's in a single-domain format (Conrath et al., 2003). Both domains are composed of four conserved sequence stretches, the framework regions, surrounding three hypervariable regions, the complementarity determining regions (CDR) (Fig. 1B). The $\mathrm{VH}$ and $\mathrm{VHH}$ domains adopt an Ig fold of two $\beta$-sheets, one of four strands and one of five strands (Muyldermans et al., 2001; Padlan, 1994). Within this fold, the three hypervariable regions, clustering at one end of the domain, are located in loops that connect the $\beta$ strands where they participate in antigen recognition. The alignment of the $\mathrm{VH}$ and $\mathrm{VHH}$ amino acid sequences immediately reveals that the first and third hypervariable regions of the $\mathrm{VHH}$ are more extended than those of VH's, and in dromedary but not in llama, the CDR1 and CDR3 often contain a Cys (Conrath et al., 2003). From crystal structures of VHH we know that these Cys form a disulfide bond that possibly assists in shaping the loop structure (De Genst et al., 2006b; Muyldermans et al., 2001). Another striking distinction between $\mathrm{VH}$ and $\mathrm{VHH}$ occurs in the framework-2 region. This region is normally highly conserved in $\mathrm{VH}$ domains and populated by hydrophobic amino acids that serve as an anchoring place for the VL domain. In contrast the corresponding region in a $\mathrm{VHH}$ contains more hydrophilic amino acids (Fig. 1B), which explains the absence of VL association and the soluble behavior of $\mathrm{VHH}$ as a single-domain entity (Nguyen et al., 2001).

The paratope of a classical antibody is expected to be much larger than that of a VHH due to the presence of the three CDR's in the VL domain. However, it has been proposed that the $\mathrm{VHH}$ paratope is enlarged by an extension of the CDR1 loop (Fig. 1B) (Nguyen et al., 2000; Vu et al., 1997). Also the CDR3 loop in VHHs of dromedary (less so in llama's) contains longer loops (average 16 amino acids) than in a $\mathrm{VH}$ from human or mouse (average 14 or 12, respectively) (Muyldermans et al., 1994; Vu et al., 1997; Wu et al., 1993). Evidently, longer loop lengths (having more amino acids) increase the paratope size (Fig. 1B).

Much of the paratope diversity in classical antibodies originates from the VH-VL pairing. Such combinatorial diversification is obviously absent in the paratope of a $\mathrm{VHH}$ as there is no VL domain in HCAbs. This limitation in the paratope repertoire is compensated by the antigenbinding loops that adopt novel conformations, never observed in human or mouse VHs. Indeed, the crystal structures of VHHs reveal that the antigen-binding loops of a VHH do not follow the canonical structural restrictions, and apparently many more loop architectures can be found in VHH's than in VHs (Decanniere et al., 2000; Nguyen et al., 2000, 2001). The rationale to introduce this loop diversification, especially in the CDR1, is embedded in the germline sequences in a subtle but ingenious way. By a substitution of two Phe codons (TTY) at positions that are detrimental for the loop organization, into Tyr codons (TAY), the CDR1 in a VHH acquires two additional hotspots (TA dinucleotides) for somatic hypermutations (Conrath et al., 2003; Nguyen et al., 2000). Indeed, the amino acids at these positions in the CDR1 are much less conserved in VHH than in VH.

Probably, the impact of the CDR1 and CDR2 on the antigen recognition has to be limited since the crystal structure survey on dromedary VHH's in complex with lysozyme indicated that the CDR3 is the main contributor of antigen binding (De Genst et al., 2006b). Whereas the six loops of a VH-VL pair contribute more or less equally to antigen binding, in a VHH the CDR3 loop dominates by providing at least $60-80 \%$ of the contacts with antigen. Moreover, we noticed that the VHH accumulates many and surprisingly convergent somatic hypermutations, probably during the affinity maturation process during immunization (De Genst et al., 2004, 2005).

Apart from the increased paratope repertoire generated by a greater diversity in the architecture of antigenbinding loops, the single-domain format leaves a footprint of its paratope on the antigen that is only half the size as opposed to that of the two-domain format (VH-VL) of classical antibodies. The VHH paratope, often helped by a protruding long CDR3 loop (De Genst et al., 2006b; Desmyter et al., 1996), is also convex shaped, in contrast to the VH-VL paratope that is either forming a concave or flat surface. Consequently, it came as no surprise to find that dromedary VHH's target preferably the antigen surfaces with concave topology such as the clefts forming the active site of enzymes (Lauwereys et al., 1998). This $\mathrm{HCAb}$ bias for unique epitopes that differ from preferred epitopes of classical antibodies was supported repeatedly by the observation that VHHs often act as enzyme inhibitors (or activators) (Lauwereys et al., 1998; Transue et al., 1998; Desmyter et al., 2002; Saerens et al., 2004), a finding that was contradicted for llama HCAbs (Ferrari et al., 2007).

\section{HCAb generation}

It can be inferred, from homology with human or mouse IgH chromosome loci, that the dromedary genome contains a cluster of $\mathrm{VH}$ and $\mathrm{VHH}$ germline genes, followed by a pool of $\mathrm{D}$ genes, a pool of $\mathrm{J}$ genes and the $\mathrm{C}$ genes including $\mu, \gamma, \varepsilon, \alpha$. The presence of a $C \delta$ gene in the llama or dromedary IgH locus is uncertain, as it has been for a long time in artiodactyls (pigs) as well (Zhao et al., 2002). However, it is clear that the dromedary genome contains multiple $\mathrm{C} \gamma$ genes, some of which are dedicated to constitute the conserved part of the H-chain of HCAbs whereas other $\mathrm{C} \gamma$ genes are employed to produce the $\mathrm{H}$ - 
(A)
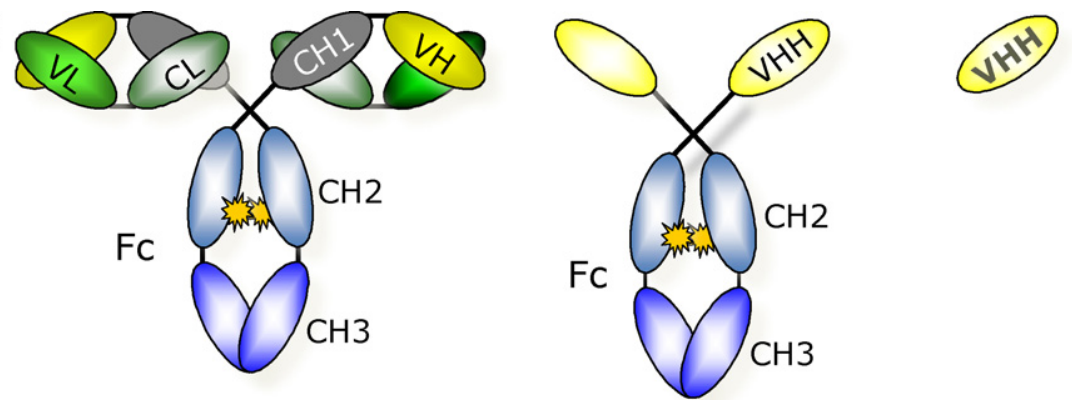

(B)

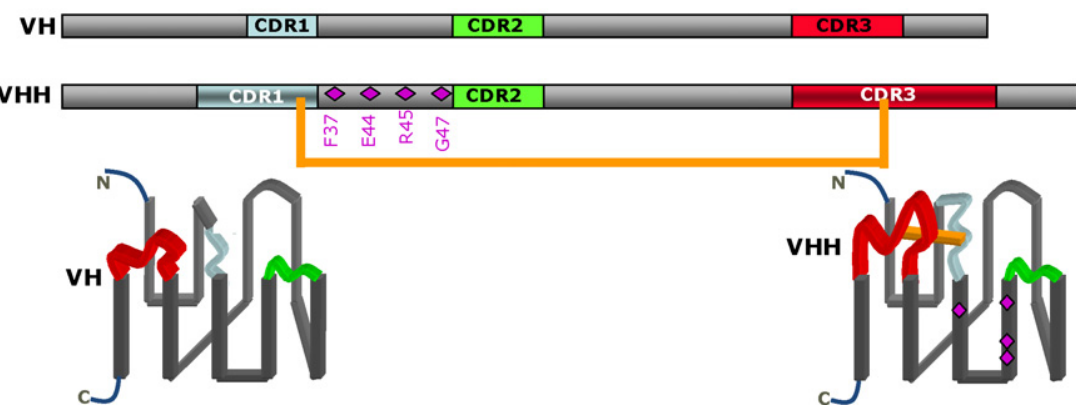

Fig. 1. (A) From left to right the composition of a classical antibody (left), a heavy-chain antibody (middle) and a single-domain antigen-binding entity derived from a heavy-chain antibody, the VHH or nanobody (right). The domain structure within the H-chain and L-chains shown. (B) The sequence organization of the VH and VHH with framework and CDR's is schematically represented at the top of this panel. The hallmark amino acids in framework-2 within the VHH are given, as well as the inter-CDR disulfide bond occurring in the dromedary VHH. Below is the folded structure of the VH (left) and VHH domain (right with its four $\beta$-stranded sheet (back) and the five $\beta$-stranded sheet in front). The hallmark VHH residues in framework- 2 are shown as squares.

chain of classical Abs (Nguyen et al., 2003; Zou et al., 2005). The dromedary genome contains about $50 \mathrm{VH}$ and $40 \mathrm{VHH}$ germline genes. The VHH germline genes have their Cys encoded in the CDR1 region, and the characteristic amino acid substitutions within the VHH-framework-2 region (Nguyen et al., 1998). The exact number of D genes is unknown, however, it seems that the J clusters comprises 5-6 genes (the most upstream ( $5^{\prime}$ located) gene might be a pseudogene) (De Genst et al., 2006a). Since the same D and $\mathrm{J}$ genes can be rearranged to either one of the $\mathrm{VH}$ or one of the VHH germline genes, it is probably safe to conclude that VH and VHH are part of the same locus (De Genst et al., 2006a; Nguyen et al., 2000). After VDJ rearrangement it seems that the Cys is introduced somatically in the CDR3 region for $\mathrm{VHH}$ but not so for $\mathrm{VH}$ domains. As it is difficult to imagine how this is accomplished during the DNA recombination, it is most likely a consequence of a subsequent selection, i.e. rearranged $\mathrm{VHH}$ regions without a Cys codon in their CDR3 will not survive the B cell selection process, or need to knock-out the Cys codon in their CDR1.

Much of our current knowledge on the human or mouse $B$ cell ontogeny and maturation came from transgenic mouse models. However, in the case of the ontogeny of B cells producing bona fide HCAbs, the data from transgenic mice yielded even conflicting results (Brüggemann et al., 2006). For example, a transgenic mice expressing the dromedary rearranged VHH-DJ joined to the HCAb dedicated $\mathrm{C} \gamma 2 \mathrm{a}$ gene produced an $\mathrm{H}$-chain transcript in which the $\mathrm{CH} 1$ exon was deleted (and consequently the $\mathrm{CH} 1$ domain in the $\mathrm{H}$-chain polypeptide as well). A single-point mutation from the conserved GT at the $5^{\prime}$ splicing site of the intron to AT was held responsible for this special VHH to hinge splicing (Zou et al., 2005). In contrast, a transgenic mouse carrying the human $\mathrm{C} \gamma$ gene with the same mutation at the splicing site failed to remove the $\mathrm{CH} 1$ exon from the transcript (Janssens et al., 2006). The current knowledge predicts that in the pre-B cell stage, the rearranged $\mathrm{H}$-chain is expressed and retained in the endoplasmic reticulum (ER) by BiP chaperones that bind at the $\mathrm{V}$ and $\mathrm{CH} 1$ domains. The two BiP chaperones per $\mathrm{H}$-chain need to be replaced by the surrogate L-chain complex to expose the pre-B cell receptor on the cell surface, to trigger the allelic exclusion, to initiate the L-chain rearrangement and to stop the expression of the VpreB and $\lambda 5$ surrogate L-chain components (Martensson et al., 2007) (Fig. 2). The functional surrogate L-chain complex consists of the VpreB- and the $\lambda 5$-associated polypeptides, whereby the $\mathrm{VpreB}$ functions as a VL substitute and the $\lambda 5$ as a CL substitute. According to our unpublished data the rearranged VHH-DJ domain is expressed with the $\mathrm{C} \mu \mathrm{H}$-chain containing the $\mathrm{CH} 1$ domain. It is difficult to envisage how this single BiP attached to this $\mathrm{C} \mu 1$ domain and retaining the $\mathrm{H}$-chain in the ER will be replaced by the surrogate L-chain complex to secrete the VHH-pre-B cell receptor (Fig. 2A). Our current working hypothesis foresees that no pre-B cell receptor can be made with the VHH-DJ in association with the $\mathrm{C} \mu$, and therefore the signal to initiate the L-chain rearrangement cannot be given. Normally, such B cells will go into apoptosis, however, in case of HCAb ontogeny, a $\mathrm{C} \mu$ to $\mathrm{C} \gamma$ class switch might be attempted for the $B$ cell to survive. In any case, after the VHH-DJ rearranged genes have been switched in the dromedary H-locus from $\mathrm{C} \mu$ to one of the $\mathrm{C} \gamma$ genes with the 

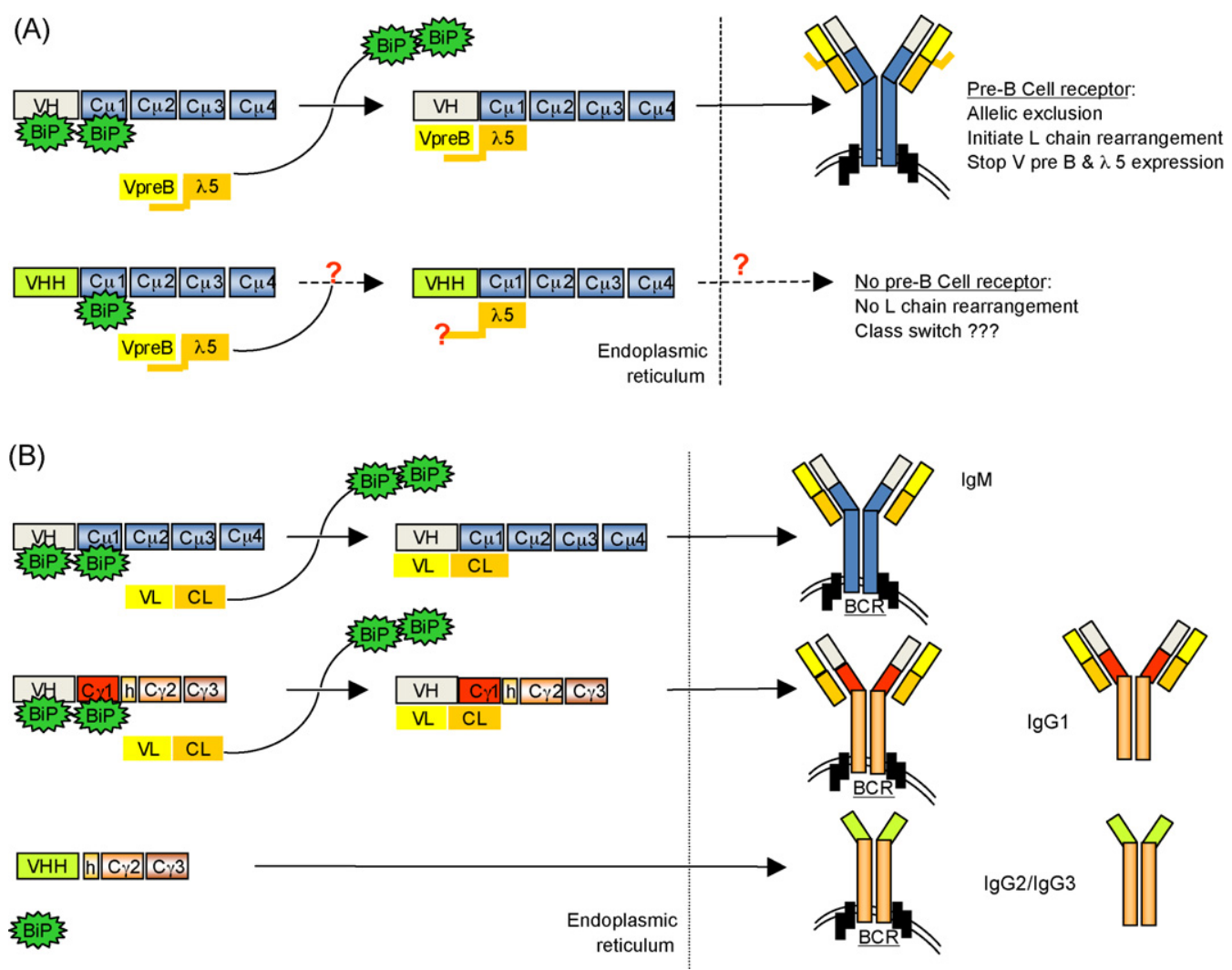

Fig. 2. (A) The translation product of the $\mathrm{VH}-\mathrm{C} \mu$ gene in the pre-B cell is kept in the endoplasmic reticulum by the BiP protein. These are replaced by the surrogate light chain (VpreB and $\lambda 5$ ) and the complex is expressed as a B cell receptor (top panel). In case of a VHH-C $\mu$ polypeptide, only the $\lambda 5$ could bind to $\mathrm{C} \mu 1$, whereas the VpreB is expected to fail to pair with the VHH domain due to the hallmark framework-2 amino acids (lower panel). (B) In the mature B cell, a VH-C $\mu$ is assembled with the rearranged L-chain and expressed on the surface of the cell as an IgM. After class switch the same VH is joined to a $\mathrm{C} \gamma$ to produce the BCR or the soluble IgG1 (middle panel). In case of a VHH-Cy expression with a $\mathrm{CH} 1$ exon deleted, we expect that a dimer of this polypeptide is immediately expressed on the B cell surface or secreted in the serum as IgG2 or IgG3 HCAbs (lower panel).

GT to AT mutation, there is apparently no further problem to produce HCAbs (Fig. 2B). The VHH domain should not be captured by the $\mathrm{BiP}$, since the $\mathrm{CH} 1$ domain is absent in the dedicated $\mathrm{C} \gamma$ genes for HCAbs due to this point mutation at the $3^{\prime}$ end consensus splicing signal of the $\mathrm{CH} 1$ exon region (Elkabetz et al., 2005; Nguyen et al., 1999; Woolven et al., 1999).

\section{Applications with Nbs}

Genetic engineering techniques allow for a fast cloning and selection of antigen-specific VHH's, referred to as Nbs. The Nbs can in theory be selected from vast, synthetic or naïve VHH libraries (Tanha et al., 2001; Verheesen et al., 2006; Yau et al., 2003), although these often recognize the antigen with low affinity, and therefore we opt for a prior immunization of a dromedary or llama as this gives us affinity-matured Nbs. Immunizing a llama or dromedary by repeated subcutaneous injections in presence of adjuvant is performed as in any other mammal (e.g. goat and rabbit). After a short immunization step (6-7 weeks) we purify the lymphocytes from a lymph node biopsy or a 50-100-ml peripheral blood sample and after an RT-PCR we clone the $\mathrm{VHH}$ repertoire from the B lymphocytes in the sample in a phage display vector (Nguyen et al., 2001; Saerens et al., 2004). The VHHs are expressed at the tip of the phage particles and after $2-3$ rounds of panning we can identify the individual clones producing virions harboring an antigen-specific VHH. This method of identifying Nbs from 'immune' libraries is much more efficient than corresponding methods to identify antigen binders from immune scFv or Fab libraries. In case of classical antibodies the $\mathrm{VH}-\mathrm{VL}$ pairs of the $\mathrm{B}$ cell receptor (BCR) are matured as a VH-VL heterodimer. During the cloning of the domains of the paratope, the VH and VL are amplified separately by PCR and joined at a later stage. This makes that for $10^{5}$ different BCR's one needs to amplify $10^{5}$ different $\mathrm{VH}$ gene regions and $10^{5}$ different $V L$ gene regions that later have to be joined in $10^{10}$ clones to encompass the entire repertoire from which to retrieve the original $\mathrm{VH}-\mathrm{VL}$ pair that matured as an entity. In contrast, for the HCAb-type BCR's of $10^{5}$ different $\mathrm{B}$ cells, one needs to clone a repertoire of $10^{5} \mathrm{VHHs}$ only as each VHH gene is comprised in one single gene fragment of $350 \mathrm{bp}$. The retrieval of antigen-specific VHHs from such (relatively) small libraries is evidently much simpler than from large libraries.

Once a clone with an antigen-specific VHH is identified, it is normally straightforward to express mg quantities of 
the soluble, properly folded $\mathrm{Nb}$ in bacterial systems, and yeasts produce even larger quantities (Frenken et al., 2000; van der Linden et al., 2000). In contrast, the expression of, e.g. scFv is routinely 10 times lower than the expression levels for Nbs. In addition, the stability of Nbs is higher compared to that of scFv independent of whether the shelf live, the thermal stability, the stability against unfolding with chemical denaturants or the protease resistance are considered (Dumoulin et al., 2002; Harmsen et al., 2006). The successful gut-passage of Nbs in a functional form was reported by Ablynx (www.ablynx.com). While the specificity and the affinity of the Nb competes easily with the better scFv or Fabs, the strict monomeric behavior and its small gene of only $350 \mathrm{bp}$ makes the Nb ideal to tailor into larger bivalent or bispecific constructs (Conrath et al., 2001).

In our laboratory and in that of others as well, the Nbs have been used as a research tool and in a variety of diagnostic or therapeutic applications. To increase the versatility of the $\mathrm{VHH}$, a stable $\mathrm{VHH}$ domain has been identified that is well expressed and acts as a loop acceptor to graft the loops of other, less stable or less well expressed, VHHs and thereby arriving at a chimeric domain with an enhanced stability, better expression and with the antigen specificity and affinity of the loop donor VHH (Saerens et al., 2005).

In another effort we have cloned the pig Fc isotypes behind a $\mathrm{Nb}$ to arrive at a chimeric HCAb composed of a VHH-pigFc (see article by Butler et al., this issue). This chimeric protein is used to generate monoclonal antibodies against pig IgG isotypes. Following the same strategy of generating genetic man-made constructs with the $\mathrm{Nb}$ gene fused to $\beta$-lactamase, or a truncated gene version of the human ApoLI or mRFP (apolipoprotein L-I or monomeric red fluorescent protein, respectively) we obtained interesting constructs for therapeutic-, or research purposes (Baral et al., 2006; Cortez-Retamozo et al., 2004; Rothbauer et al., 2006). The lactamase fusion construct comprised a $\mathrm{Nb}$ directed against carcino-embryonic antigen (CEA), a human tumor marker. Mice carrying a human tumor were injected with this protein fusion to load the tumor with the conjugate. A prodrug administered in the vein of the mice will be converted to a highly toxic molecule by the lactamase. Consequently, a high concentration of the toxin is produced, mainly in the vicinity of the tumor site. Following this antibody-dependent enzyme prodrug therapy approach, we could eradicate - entirely and without relapse - the established solid tumors in mouse models (Cortez-Retamozo et al., 2004).

With the construct of the truncated ApoLI attached to a $\mathrm{Nb}$ that recognizes the Trypanosome rhodesiensis, a parasite that causes sleeping sickness in East Africa, we generated a strong trypanolytic compound that eliminated the parasites from blood (Baral et al., 2006; Stijlemans et al., 2004). This Nb recognizes the conserved carbohydrate of the trypanosomal Variant surface glycoprotein, the major coat protein that covers the entire surface of the parasite and which is exchanged by one of its variants during the antigenic variation, the main parasite mechanism to escape from the host immune response.
Finally, extending the Nb with the MRFP (referred to as a chromobody) generated a useful tool to target and to uncover the residence of antigen in living cells. Proof of principle was given with Nbs directed against cytokeratine and lamin (Rothbauer et al., 2006). Cell lines, transiently transfected with the genetic constructs of the respective Nb-mRFP revealed under the confocal microscope convincing pictures where the antigens were stained with the chromobodies. In addition, it has been proved recently that Nbs tethered to a solid support are ideal to capture their cognate antigen even when present at low doses in a complex mixture (Rothbauer et al., 2008). Hence, we can recommend the $\mathrm{Nb}$ as a highly antigen-specific tool for immunoprecipitation, although in Western blots its performance is rather poor.

In conclusion, antigen-specific Nbs are readily identified after a short immunization of a llama, a camel or a dromedary with antigen, a cloning step of the VHH repertoire from the immunized animal and phage display. The good expression in microbial systems and the beneficial biochemical properties (good solubility, good stability in harsh conditions, high affinity and specificity for the antigen, small size and strict monomeric behavior) make that Nbs are an ideal tool for research purposes or diagnostic or therapeutic applications.

\section{Conflict of interest}

None.

\section{Acknowledgements}

D.S. receives a FWO postdoc fellowship, and S.T. received a DWTC scholarship. The authors thank the financial support of their research through grants from EU, FWO, IWT and OZR-VUB.

\section{References}

Baral, T.N., Magez, S., Stijlemans, B., Conrath, K., Vanhollebeke, B., Pays, E., Muyldermans, S., De Baetselier, P., 2006. Experimental therapy of African trypanosomiasis with a nanobody-conjugated human trypanolytic factor. Nat. Med. 12, 580-584.

Brüggemann, M., Smith, J.A., Osborn, M., Corcos, D., Zou, X., Nguyen, V.K., Muyldermans, S., 2006. Heavy chain only antibody expression and Bcell development in the mouse. Crit. Rev. Immunol. 26, 377-390.

Conrath, K.E., Lauwereys, M., Wyns, L., Muyldermans, S., 2001. Camel single-domain antibodies as modular building units in bispecific and bivalent antibody constructs. J. Biol. Chem. 276, 7346-7350.

Conrath, K.E., Wernery, U., Muyldermans, S., Nguyen, V.K., 2003. Emergence and evolution of functional heavy-chain antibodies in Camelidae. Develop. Comp. Immunol. 27, 87-103.

Cortez-Retamozo, V., Backmann, N., Senter, P., Wernery, U., De Baetselier, P., Muyldermans, S., Revets, H., 2004. Efficient cancer therapy with a nanobody-based conjugate. Cancer Res. 64, 2853-2857.

De Genst, E., Handelberg, F., Van Meirhaeghe, A., Vinck, S., Loris, R., Wyns, L., Muyldermans, S., 2004. Chemical basis for the affinity maturation of a camel single domain antibody. J. Biol. Chem. 279, 53593-53601.

De Genst, E., Saerens, D., Muyldermans, S., Conrath, K., 2006a. Antibody repertoire development in camelids. Develop. Comp. Immunol. 30, 187-198.

De Genst, E., Silence, K., Decanniere, K., Conrath, K., Loris, R., Kinne, J., Muyldermans, S., Wyns, L., 2006b. Molecular basis for the preferential cleft recognition by dromedary heavy-chain antibodies. Proc. Natl. Acad. Sci. U.S.A. 103, 4586-4591.

De Genst, E., Silence, K., Ghahroudi, M.A., Decanniere, K., Loris, R., Kinne, J., Wyns, L., Muyldermans, S., 2005. Strong in vivo maturation compen- 
sates for structurally restricted $\mathrm{H} 3$ loops in antibody repertoires. J. Biol. Chem. 280, 14114-14121.

Decanniere, K., Muyldermans, S., Wyns, L., 2000. Canonical antigen binding loop structures: more structures, more canonical classes? J. Mol. Biol. 300, 83-91.

Desmyter, A., Spinelli, S., Payan, F., Lauwereys, M., Wyns, L., Muyldermans, S., Cambillau, C., 2002. Three camelid VHH domains in complex with porcine pancreatic á-amylase: inhibition and versatility of binding topology. J. Biol. Chem. 277, 23645-23650.

Desmyter, A., Transue, T.R., Arbabi Ghahroudi, M., Dao-Thi, M.-H., Poortmans, F., Hamers, R., Muyldermans, S., Wyns, L., 1996. Crystal structure of a camel single-domain $\mathrm{VH}$ antibody fragment in complex with lysozyme. Nat. Struct. Biol. 3, 803-811.

Dumoulin, M., Conrath, K., Van Meirhaeghe, A., Meersman, F., Heremans, K., Frenken, L.G., Muyldermans, S., Wyns, L., Matagne, A., 2002. Singledomain antibody fragments with high conformational stability. Protein Sci. 11, 500-515.

Elkabetz, Y., Argon, Y., Bar-Nun, S., 2005. Cysteines in CH1 underlie retention of unassembled Ig heavy chains. J. Biol. Chem. 280, 14402-14412.

Ferrari, A., Rodriguez, M.M., Power, P., Weill, F.S., De Simone, E.A., Gutkind, G., Leoni, J., 2007. Immunobiological role of llama heavy-chain antibodies against a bacterial $\beta$-lactamase. Vet. Immunol. Immunopathol. 117, 173-182.

Frenken, L., van der Linden, R., Hermans, P.W.J.J., Bos, W., Ruuls, R.C., de Geus, B., Verrips, T., 2000. Isolation of antigen specific llama VHH antibody fragments and their high level secretion by Saccharomyces cerevisiae. J. Biotechnol. 78, 11-21.

Hamers-Casterman, C., Atarhouch, T., Muyldermans, S., Robinson, G., Hamers, C., Bajyana Songa, E., Bendahman, N., Hamers, R., 1993. Naturally occurring antibodies devoid of light chains. Nature 363, 446-448.

Harmsen, M.M., Ruuls, R.C., Nijman, I.J., Niewold, T.A., Frenken, L., de Geus, B., 2001. Llama heavy chain V-regions consist of at least four distinct subfamilies revealing novel sequence features. Molec. Immunol. 37, 579-590.

Harmsen, M.M., van Solt, C.B., van Zijderveld-van Bemmel, A.M., Niewold, T.A., van Zijderveld, F.G., 2006. Selection and optimization of proteolytically stable llama single-domain antibody fragments for oral immunotherapy. Appl. Microbiol. Biotechnol. 72, 544-551.

Janssens, R., Dekker, S., Hendriks, R.W., Panayotou, G., van Remoortere, A. San, J.K., Grosveld, F., Drabek, D., 2006. Generation of heavy-chain-only antibodies in mice. Proc. Natl. Acad. Sci. U.S.A. 103, 15130-15135.

Lauwereys, M., Ghahroudi, M.A., Desmyter, A., Kinne, J., Hölzer, W., De Genst, E., Wyns, L., Muyldermans, S., 1998. Potent enzyme inhibitors derived from dromedary heavy-chain antibodies. EMBO J. 17, 35123520.

Maass, D.R., Sepulveda, J., Pernthaner, A., Shoemaker, C.B., 2007. Alpaca (Lama pacos) as a convenient source of recombinant camelid heavy chain antibodies (VHHs). J. Immunol. Methods 324, 13-25.

Martensson, I.L., Keenan, R.A., Licence, S., 2007. The pre-B-cell receptor. Curr. Opin. Immunol. 19, 137-142.

Muyldermans, S., Atarhouch, T., Saldanha, J., Barbosa, J.A.R.G., Hamers, R., 1994. Sequence and structure of VH domain from naturally occurring camel heavy chain immunoglobulins lacking light chains. Protein Eng. 7. 1129-1135.

Muyldermans, S., Cambillau, C., Wyns, L., 2001. Recognition of antigens by single-domain antibody fragments: the superfluous luxury of paired domains. TIBS 26, 230-235.

Muyldermans, S., Lauwereys, M., 1999. Unique single-domain antigen binding fragments derived from naturally occurring camel heavychain antibodies. J. Mol. Recognit. 12, 1-10.

Nguyen, V.K., Desmyter, A., Muyldermans, S., 2001. Functional heavychain antibodies in Camelidae. Adv. Immunol. 79, 261-296.

Nguyen, V.K., Hamers, R., Wyns, L., Muyldermans, S., 1999. Loss of splice consensus signal is responsible for the removal of the entire $\mathrm{CH} 1$ domain of the functional camel IgG2A heavy chain antibodies. Mol. Immunol. 36, 515-524.
Nguyen, V.K., Hamers, R., Wyns, L., Muyldermans, S., 2000. Camel heavychain antibodies: diverse germline VHH and specific mechanisms enlarge the antigen-binding repertoire. ЕMBO J. 19, 921-931.

Nguyen, V.K., Muyldermans, S., Hamers, R., 1998. The specific variable domain of camel heavy-chain antibodies is encoded in the germline. J. Mol. Biol. 257, 413-418.

Nguyen, V.K., Zou, X., Lauwereys, M., Brys, L., Brüggemann, M., Muyldermans, S., 2003. Heavy-chain only antibodies derived from dromedary are secreted and displayed by mouse B cells. Immunology 109, 93101.

Padlan, E.A., 1994. Anatomy of the antibody molecule. Molec. Immunol. 31, 169-217.

Rothbauer, U., Zolghadr, K., Muyldermans, S., Schepers, A., Cardoso, C.M., Leonhardt, H., 2008. A versatile nanotrap for biochemical and functional studies with fluorescent fusion proteins. Mol. Cell. Proteomics 7, 282-289.

Rothbauer, U., Zolghadr, K., Tillib, S., Nowak, D., Schermelleh, L., Gahl, A., Backmann, N., Conrath, K., Muyldermans, S., Cardoso, C.M., Leonhardt, H., 2006. Targeting and tracing antigens in live cells with fluorescent nanobodies. Nat. Methods 3, 887-889.

Saerens, D., Kinne, J., Bosmans, E., Wernery, U., Muyldermans, S., Conrath, K., 2004. Single domain antibodies derived from dromedary lymph node and peripheral blood lymphocytes sensing conformational variants of prostate-specific antigen. J. Biol. Chem. 279, 5196551972.

Saerens, D., Pellis, M., Loris, R., Pardon, E., Dumoulin, M., Matagne, A., Wyns, L., Muyldermans, S., Conrath, K., 2005. Identification of a universal framework to graft non-canonical antigen binding loops of camel single domain antibodies. J. Mol. Biol. 352, 597-607.

Stijlemans, B., Conrath, K., Cortez-Retamozo, V., Van Xong, H., Wyns, L., Senter, P.D., Revets, H., De Baetselier, P., Muyldermans, S., Magez, S., 2004. Efficient targeting of conserved cryptic epitopes of infectious agents by single-domain antibodies: African trypanosomes as paradigm. J. Biol. Chem. 279, 1256-1261.

Tanha, J., Xu, P., Chen, Z., Ni, F., Kaplan, H., Narang, S.A., MacKenzie, R., 2001. Optimal design features of camelized human single domain antibody libraries. J. Biol. Chem. 276, 24774-24780.

Transue, T.R., De Genst, E., Ghahroudi, M.A., Wyns, L., Muyldermans, S., 1998. Camel single domain antibody inhibits enzyme by mimicking carbohydrate substrate. Proteins: Struct. Funct. Genetics 32, 515-522.

van der Linden, R.H.J., de Geus, B., Frenken, L., Peters, H., Verrips, T., 2000. Improved production and function of llama heavy chain antibody fragments by molecular evolution. J. Biotechnol. 80, 261-270.

Verheesen, P., Roussis, A., de Haard, H.J., Groot, A.J., Stam, J.C., den Dunnen, J.T., Frants, R.R., Verkleij, A.J., Verrips, T.C., van der Maarel, S.M., 2006. Reliable and controllable antibody fragment selections from Camelid non-immune libraries for target validation. Biochim. Biophys. Acta $1764,1307-1319$.

Vu, K.B., Ghahroudi, M.A., Wyns, L., Muyldermans, S., 1997. Comparison of llama VH sequences from conventional and heavy chain antibodies. Mol. Immunol. 34, 1121-1131.

Woolven, B.P., Frenken, L., van der Logt, P., Nicholls, P.J., 1999. The structure of the llama heavy chain constant genes reveals a mechanism for heavy-chain antibody formation. Immunogenetics 50, 98101.

Wu, T.T., Johnson, G., Kabat, E.A., 1993. Length distribution of CDR H3 in antibodies. Proteins: Struct. Funct. Genetics 16, 1-7.

Yau, K.Y.F., Groves, M.A.T., Li, S., Sheedy, C., Lee, H., Tnaha, J., MacKenzie, C.R., Jermutus, L., Hall, J.C., 2003. Selection of hapten-specific singledomain antibodies from a non-immunized llama ribosome display library. J. Immunol. Methods 281, 161-175.

Zhao, Y., Kacskovics, I., Pan, Q., Liberles, D.A., Geli, J., Davis, S.K., Rabbani, H., Hammarstrom, L., 2002. Artiodactyl IgD: the missing link. J. Immunol. 169, 4408-4416.

Zou, X., Smith, J.A., Nguyen, V.K., Ren, L., Luyten, K., Muyldermans, S., Brüggemann, M., 2005. Expression of a dromedary heavy-chain only antibody and B cell development in the mouse. J. Immunol. 175, 3769-3779. 